\title{
B7-H3 promotes the epithelial-mesenchymal transition of NSCLC by targeting SIRT1 through the PI3K/AKT pathway
}

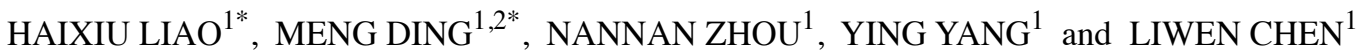 \\ ${ }^{1}$ Department of Laboratory Medicine, The Second Hospital of Anhui Medical University, Hefei, Anhui 230601; \\ ${ }^{2}$ Department of Laboratory Medicine, The Second Hospital of Nanjing, \\ Nanjing University of Chinese Medicine, Nanjing, Jiangsu 210003, P.R. China
}

Received September 30, 2021; Accepted December 13, 2021

DOI: $10.3892 / \mathrm{mmr} .2022 .12595$

\begin{abstract}
Epithelial-mesenchymal transition (EMT) is a key step in cancer metastasis. B7-H3, a co-signaling molecule associated with poor prognosis of non-small cell lung cancer (NSCLC), promotes the metastasis of NSCLC by activating the EMT process. However, its underlying mechanism remains poorly understood. In the present study, it was shown that CRISPR/Cas9-mediated B7-H3 deletion downregulated the expression of the class III histone deacetylase, sirtuin-1 (SIRT1), in NSCLC A549 cells. Accordingly, SIRT1 silencing resulted in markedly decreased migration and invasion of A549 cells. Both B7-H3 gene-edited and SIRT1-silenced cells were typically characterized by an increased expression of the epithelial marker E-cadherin, and downregulation of the mesenchymal markers $\mathrm{N}$-cadherin and vimentin, as compared with mock-edited and scrambled negative small interfering RNA control, respectively. It was further demonstrated that B7-H3 ablation significantly downregulated phosphorylated AKT/protein kinase B expression, and SIRT1 expression was substantially suppressed by the PI3K-specific inhibitor, LY294002. Taken together, the findings of the present study revealed that B7-H3-induced signaling upregulates SIRT1 expression via the PI3K/AKT pathway to promote EMT activation that is associated with metastasis in NSCLC.
\end{abstract}

Correspondence to: Dr Liwen Chen, Department of Laboratory Medicine, The Second Hospital of Anhui Medical University, 678 Furong Road, Hefei, Anhui 230601, P.R. China

E-mail: chenliwen@ahmu.edu.cn

*Contributed equally

Abbreviations: EMT, epithelial-mesenchymal transition; NSCLC, non-small cell lung cancer; SCLC, small cell lung cancer; SIRT1, Sirtuin 1; AKT/PKB, Protein kinase B; PI3K, phosphoinositide 3kinase; $\mathrm{KO}$, knockout; mock, negative control; siControl, negative control siRNAs oligos; DMSO, dimethyl sulfoxide; t-, total; p-, phosphorylated; ECL, enhanced chemiluminescent; HRP, horseradish peroxidase

Key words: NSCLC, epithelial-mesenchymal transition, migration, invasion, B7-H3

\section{Introduction}

Globally, the estimated statistics for the year 2018 relating to 36 different types of cancer in 185 countries revealed that lung cancer remains the leading cause of cancer incidence and mortality, with reported percentages of $11.6 \%$ of the total cases and $18.4 \%$ of the total cancer deaths, respectively (1). Histological classification distinguishes two main subtypes of lung cancer: Small cell lung cancer (SCLC) and non-SCLC (NSCLC). The latter has an incidence rate exceeding $85 \%$ (2). Metastases are a frequent complication of NSCLC, which most commonly spreads to the bones, brain, liver and adrenal glands (2). As the majority of patients with metastatic NSCLC are inoperable at the time of diagnosis, the prognosis is generally poor, with a 5-year survival rate of $<20 \%$ (3).

Epithelial-mesenchymal transition (EMT) is an evolutionarily conserved developmental program that confers metastatic properties to malignancies by enhancing the adhesion, migration and invasion of cancer cells $(4,5)$. The EMT encompasses dynamic changes in cellular organization, leading to the loss of epithelial markers and the gain of mesenchymal phenotypes. E-cadherin is among the most important molecules that regulate cell-cell adhesion in epithelial tissues. By contrast, $\mathrm{N}$-cadherin and vimentin are mesenchymal hallmarks that represent the acquisition of an aggressive tumor phenotype (6). Sirtuin-1 (SIRT1) is also an evolutionarily conserved, $\mathrm{NAD}^{+}$-dependent class III histone deacetylase that plays a key role in epigenetic regulation by deacetylating both histone and non-histone targets (7). SIRT1 has gained significant interest due to its ability to promote genomic stability and metabolic regulation; however, mounting evidence has suggested an oncogenic role for SIRT1 (8). Particularly, SIRT1 is associated with tumor metastasis via the positive regulation of EMT, promoting the migratory capability of cancer cells in vitro and tumor metastasis in vivo (9).

B7-H3 (CD276), a member of the B7 superfamily, is upregulated on different types of malignant cells, including NSCLC cells (10-13). The membrane-bound B7-H3 not only inhibits tumor-specific $\mathrm{T}$ cell activation $(14,15)$, but it also transduces intracellular signals to promote tumor cell migration and invasion, angiogenesis, drug resistance and the Warburg effect (16-21). Therefore, B7-H3 may act as a novel target for cancer immunotherapy (17-22). B7-H3 has been shown to 
promote the EMT process of NSCLC cells (16); however, the underlying signaling mechanisms have yet to be fully elucidated. Therefore, the aim of the present study was to analyze the potential role exerted by SIRT1 in B7-H3-mediated EMT, and the possible mechanisms through which B7-H3-induced signaling may regulate SIRT1 expression in NSCLC A549 cells.

\section{Materials and methods}

Cell lines and cell culture. The A549 NSCLC cell line (cat. no. FH0045) was obtained from the Cell Culture Center of FuHeng Biology, and the A549 cells were maintained in Invitrogen ${ }^{\circledR}$ RPMI-1640 medium (Thermo Fisher Scientific, Inc.) supplemented with $10 \%$ FBS (Lonsera; Shanghai Shuangru Biotechnology Co., Ltd.) and penicillin (100 IU/ml)/streptomycin $(100 \mu \mathrm{g} / \mathrm{ml})$ (MedChemExpress). The A549 cell line was authenticated by using short tandem repeat (STR) analysis in combination with sex-typing gene amelogenin detection, and the A549 cell line was compared with the DSMZ STR cell line profile before use. The cells were incubated in a humidified atmosphere containing $5 \% \mathrm{CO}_{2}$ at $37^{\circ} \mathrm{C}$.

Genome editing of B7-H3 and gene silencing of SIRT1. Knockout (KO) of the $B 7-H 3$ gene in the A549 cell line was performed using CRISPR/Cas9 guide constructs, on the basis of a previously published protocol (23). The sequence of single guide RNA (sgRNA) was TTGATGTGCACAGCGTCCTGC GG, which was designed by using the online tool available from ZHANG LAB (https://zlab.bio/guide-design-resources). The target sequences for sgRNA were the N-terminal $250 \mathrm{bp}$ of exon 4 (315 bp), which encode the transmembrane domain of B7-H3 . The sgRNA-expressing plasmid was constructed by annealing the custom sgRNA (Sangon Biotech Co., Ltd.) to a lentiviral vector U6-MCS-sgRNA-SV40-EGFP (cat. no. GV504) developed by Genechem, Inc. The lentivirus expressing only Cas9 was used to generate negative control (mock) KO cells. SIRTl knockdown was performed by transfection of specific small interfering RNAs (siRNAs) using Lipofectamine ${ }^{\circledR} 3000$ (Thermo Fisher Scientific, Inc.) according to the manufacturer's protocol. The sequences of the on-target siRNAs were as follows: siSIRT1 \#1, 5'-GCGGGAAUCCAAAGGAUA ATT-3' and siSIRT1 \#2, 5'-UUAUCCUUUGGAUUCCCG CTT-3'. Those of the negative control siRNAs oligos were: siControl \#1, 5'-UUCUCCGAACGUGUCACGUTT-3' and siControl \#2, 5'-ACGUGACACGUUCGGAGAATT-3'. All the oligos were purchased from Shanghai GenePharma Co., Ltd. The final concentration of Lipofectamine 3000 and siRNAs were $2 \mu \mathrm{l} / \mathrm{ml}$ and $50 \mathrm{nM}$, respectively. The siRNA-transfected A549 cells were incubated at $37^{\circ} \mathrm{C}$ in $5 \% \mathrm{CO}_{2}$, and SIRT1 knockdown was verified at $48 \mathrm{~h}$ post-transfection via western blotting, as described below.

Cell proliferation assay. B7-H3 KO and mock A549 cells were cultured for $0,24,48$ and $72 \mathrm{~h}$, and the fold-change of cell proliferation was assayed by quantitation of the uptake and digestion of WST-8/Cell Counting Kit-8 (CCK-8) according to the manufacturer's instructions (Dojindo Laboratories, Inc.). Cell proliferation at $0 \mathrm{~h}$ was normalized to 1.0 . All experiments were performed in triplicate.
Western blot analysis. B7-H3 KO and mock-edited, siSIRT1 and siControl A549 cells were left untreated, whereas the wild-type A549 cells were either treated with the PI3K-specific inhibitor, LY294002 (50 $\mu \mathrm{M}$; MedChemExpress), or DMSO control at $37^{\circ} \mathrm{C}$ for $24 \mathrm{~h}$. Cells were lysed with a radio-immunoprecipitation assay (RIPA) buffer containing $1 \mathrm{mM}$ protease inhibitor phenylmethylsulfonyl fluoride (PMSF) (cat. no. R0020; Beijing Solarbio Science \& Technology Co., Ltd.), and $1 \%$ (v/v) protein phosphatase inhibitor (All-in-one) mixture (cat. no. P1260; Beijing Solarbio Science \& Technology Co., Ltd.). The total cell lysate was measured by bicinchoninic acid (BCA) assay and $30 \mu \mathrm{g}$ protein was separated via SDS-PAGE (12\% running, 5\% stacking). The separated proteins were then transferred to a polyvinylidene difluoride (PVDF) membrane, blocked with 5\% (w/v) skimmed milk in Tris-HCL buffer solution (TBS) for $2 \mathrm{~h}$ at room temperature, and incubated at $4^{\circ} \mathrm{C}$ overnight with primary antibodies against human B7-H3/CD276 (cat. no. ab227670, 1:100), E-cadherin (cat. no. ab40772, 1:10,000), N-cadherin (cat. no. ab76011, 1:5,000), vimentin (cat. no. ab92547, 1:1,000), total (t)-ERK1/2 (cat. no. ab54230, 1:1,000), phosphorylated (p)-ERK1/2 (T202+T204; cat. no. ab214362, 1:1,000) and t-STAT-3 (cat. no. ab109085, 1:5,000) (all these antibodies were purchased from Abcam); or with anti-p-STAT-3 (Tyr705; cat. no. AF3293, 1:1,000), anti-GAPDH (cat. no. AF7021, 1:3,000) (both from Affinity Biosciences, Ltd.), anti-SIRT1 (cat. no. 2493, 1:1,000), anti-t-AKT (cat. no. 4691, 1:1,000) and anti-p-AKT (Ser473; cat. no. 4060, 1:2,000) (the latter three antibodies were purchased from Cell Signaling Technology, Inc.). Subsequently, HRP-conjugated goat anti-rabbit IgG $(\mathrm{H}+\mathrm{L})$ (cat. no. S0001, 1:5,000; Affinity Biosciences, Ltd.) was used as the secondary antibody and incubated for $1 \mathrm{~h}$ at room temperature in the dark. The membranes were washed four times with TBS with $0.1 \%(\mathrm{v} / \mathrm{v})$ Tween-20 and then incubated with ECL substrate (Thermo Fisher Scientific, Inc.), and visualized using the Tanon Fine-Do X6 Chemi-Image System (Tanon Science and Technology Co., Ltd.). The expression levels of the signaling proteins were assessed using ImageJ software (v1.8.0; National Institutes of Health), and were normalized against GAPDH. All experiments were performed in triplicate.

Reverse transcription-quantitative PCR (RT-qPCR). Total RNA was isolated from $B 7-H 3 \mathrm{KO}$ and mock, and SIRTI silencing and siControl A549 cells with RNAiso Plus (Takara Bio, Inc.) according to the manufacturer's instructions. Next, cDNA was synthesized using PrimeScript RT Master Mix reverse transcription kit (Takara Bio, Inc.), according to the manufacturer's instructions, and qPCR was performed with QuantStudio 5 (ThermoFisher Scientific,Inc.) by using TaqMan gene expression assay kit/probe sets (Thermo Fisher Scientific, Inc.), according to the manufacturer's protocols. The primers used in this study were as follows: B7-H3 forward (F), 5'-AGG GCAGCCTATGACATTCCC-3' and reverse (R), 5'-AGCTCC TGCATTCTCCTCCTC A-3'; SIRT1 F, 5'-ACCTTCTGT TCGGTGATG-3' and R, 5'-TATGGACCTATCCGTGGC-3'; and $\beta$-actin F, 5'-GGAAGGTGAAGGTCGGAGTC-3' and R, 5'-CGTTCTCAGCCTTGACGGT-3'. The thermocycling conditions included an initial denaturation at $94^{\circ} \mathrm{C}$ for $10 \mathrm{~min}$; followed by 40 cycles of denaturation at $95^{\circ} \mathrm{C}$ for $15 \mathrm{sec}$, 
annealing at $55^{\circ} \mathrm{C}$ for $15 \mathrm{sec}$ and extension at $72^{\circ} \mathrm{C}$ for $1 \mathrm{~min}$. The relative expression level of B7-H3 and SIRT1 mRNA was normalized against $\beta$-actin expression and calculated using the $2^{-\Delta \Delta C q}$ method (24).

Cell migration and invasion assays. For the cell migration assay, $5 \times 10^{4}$ A549 cells from different groups were resuspended in $200 \mu 1$ serum-free medium and placed in the upper Transwell chamber (Corning, Inc.), and $600 \mu 1$ medium containing $30 \%$ FBS was added to the lower chamber. Cells were incubated at $37^{\circ} \mathrm{C}$ in an atmosphere of $5 \% \mathrm{CO}_{2}$, and allowed to migrate for $24 \mathrm{~h}$. Subsequently, the migrated cells on the lower surface of the membrane were fixed with $4 \%(\mathrm{w} / \mathrm{v})$ paraformaldehyde in PBS buffer for $40 \mathrm{~min}$ and stained with $0.1 \%$ crystal violet for $15 \mathrm{~min}$ (both at room temperature). The migrated cells were then counted, and images were captured at a magnification of $\mathrm{x} 100$ using a light microscope (Olympus Corporation). In total, five random fields of the membrane in each well were counted, and the average number/field from triplicate wells was plotted. For the cell invasion assay, a Transwell chamber (Corning, Inc.) was pre-coated with $50 \mu 1$ Matrigel matrix (BD Biosciences) in serum-free medium for $3 \mathrm{~h}$ at $37^{\circ} \mathrm{C}$ and $5 \% \mathrm{CO}_{2}$, and $1 \times 10^{5}$ A549 cells from the different treatment groups were placed in the upper chamber. After $48 \mathrm{~h}$, the lower surface of the membrane was fixed with $4 \%$ (w/v) paraformaldehyde, stained with $0.1 \%$ crystal violet and counted as described above for the cell migration assay. These experiments were performed three times.

Statistical analysis. A549 cells in the different experimental treatment groups (B7-H3 KO, siSIRT1 or LY294002-treated) were compared with the corresponding control groups, where indicated. The results are presented as the mean \pm SEM of three representative experiments, where the significance was calculated using an unpaired two-tailed Student's t-test. Statistical analyses were performed using SPSS software version 20.0 (IBM Corp.). $\mathrm{P}<0.05$ was considered to indicate a statistically significant difference.

\section{Results}

B7-H3 ablation reduces the proliferation, migration and invasion of A549 cells. To determine the potential mechanisms via which B7-H3 could promote metastasis in NSCLC, A549 cells were genome-edited to generate a $B 7-H 3 \mathrm{KO}$ using CRISPR/Cas9 technology. Subsequently, a stable $B 7-H 3 \mathrm{KO}$ A549 strain was established, and confirmed via western blotting and RT-qPCR (P<0.001; Fig. 1A). Morphologically, the B7-H3-deleted cells were characterized by a bright outline, with a round blunt shape (Fig. 1B). Furthermore, the KO of B7-H3 expression led to significantly decreased proliferation of A549 cells at 24,48 and $72 \mathrm{~h}$ after plating $(\mathrm{P}<0.001$; Fig. $1 \mathrm{C})$.

Next, a Boyden chamber assay was then used to evaluate the in vitro migratory and invasive capabilities of the $B 7-H 3$ KO A549 cells. As shown in Fig. 1D and E, B7-H3 ablation reduced the percentages of migrating and invasive cells by $>50 \%(\mathrm{P}=0.0003)$ and $70 \%(\mathrm{P}=0.0002)$, respectively, compared with the mock control. These results suggested that B7-H3 is associated with the fast-growing and aggressive status of NSCLC by promoting the proliferation and metastasis of cancer cells.

B7-H3 deletion regulates the expression of EMT-associated factors. EMT is highly dysregulated in malignancy, leading to functional changes in tumor cell migration and invasion. Consequently, the effects of $B 7-H 3 \mathrm{KO}$ on aspects typically associated with EMT were studied, including the epithelial marker E-cadherin and the mesenchymal phenotypic markers, vimentin and $\mathrm{N}$-cadherin. $\mathrm{B} 7-\mathrm{H} 3$ deletion was found to elicit a significant suppression of $\mathrm{N}$-cadherin and vimentin expression (both $\mathrm{P}<0.0001$ ), whereas the expression of E-cadherin was significantly increased compared with the mock control A549 cells $(\mathrm{P}=0.0041)$ (Fig. 2). These results further demonstrated that $B 7-H 3$ silencing decreases the EMT process, hindering the loss of adhesive junctions and the gain of mesenchymal activities.

SIRT1 is involved in the B7-H3-induced EMT process. SIRT1 is a NAD ${ }^{+}$-dependent, class III histone deacetylase involved in the epigenetic regulation of tissue homeostasis and numerous diseases, including tumorigenesis (7). SIRT1 dysregulation has already been demonstrated in various cancer cells $(8,9)$; however, its precise role in cancer development has yet to be fully elucidated. In the present study, it was first demonstrated that $B 7-H 3$ ablation led to a significant downregulation of SIRT1 expression compared with the mock control $(\mathrm{P}=0.0012$; Fig. 3A). Subsequently, the effects of SIRT1 on biological processes relevant to metastatic activity, migration and invasion were studied in A549 cells transfected with either siSIRT1 or the scrambled siControl (Fig. 3B). As shown in Fig. 3C and D, SIRT1 silencing produced effects that were similar to those of $B 7-H 3$ deletion in A549 cells, reducing the numbers of migrating and invasive cells by $>50 \%(\mathrm{P}<0.0001)$ and $60 \%(\mathrm{P}=0.0002)$, respectively, compared with the mock siControl. Consistent with these findings, SIRT1 silencing also led to a significant decrease in the expression levels of vimentin $(\mathrm{P}=0.0131)$ and $\mathrm{N}$-cadherin $(\mathrm{P}<0.0001)$, whereas $\mathrm{E}$-cadherin was observed to be upregulated $(\mathrm{P}<0.0001)$ in comparison with the scrambled control (Fig. 4). Therefore, these results suggested that SIRT1 was involved in the B7-H3-induced EMT process in A549 cells.

B7-H3 regulates SIRTl via the PI3K/AKT pathway. The PI3K/AKT, JAK2/STAT3 and Raf/MEK/ERK1/2 signaling cascades have been reported to be involved in B7-H3-induced signaling in different types of cancer cells (17-21). In view of this, the underlying mechanisms governing how B7-H3-induced signaling may regulate SIRT1 expression was investigated in the present study. First, the putative functional signaling pathway in A549 cells was assessed. Western blot analysis revealed that the expression level of p-AKT, but not of p-STAT3 or p-ERK $1 / 2$, was significantly downregulated in $B 7-H 3 \mathrm{KO}$ A549 cells compared with the mock control $(\mathrm{P}=0.0005)$ (Fig. 5A and B). Secondly, to explore whether the $\mathrm{PI3K} / \mathrm{AKT}$ pathway was involved in B7-H3 mediated SIRT1 regulation, the specific PI3K inhibitor LY294002 was used to observe its effects on SIRT1. Western blot analysis showed that LY294002 resulted in significantly decreased expression levels of SIRT1 $(\mathrm{P}<0.0001)$ and $\mathrm{p}-\mathrm{AKT}(\mathrm{P}=0.0002)$ in $\mathrm{A} 549$ cells 
A
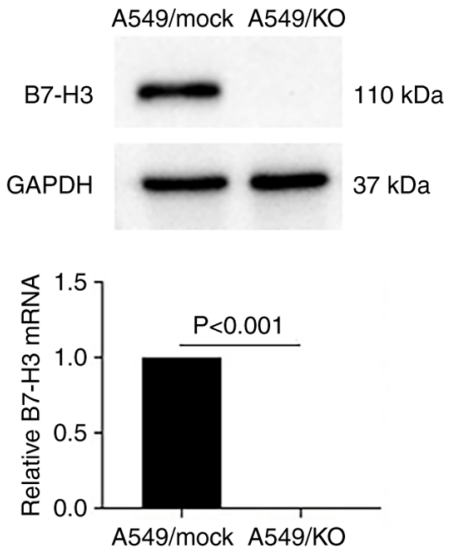

B
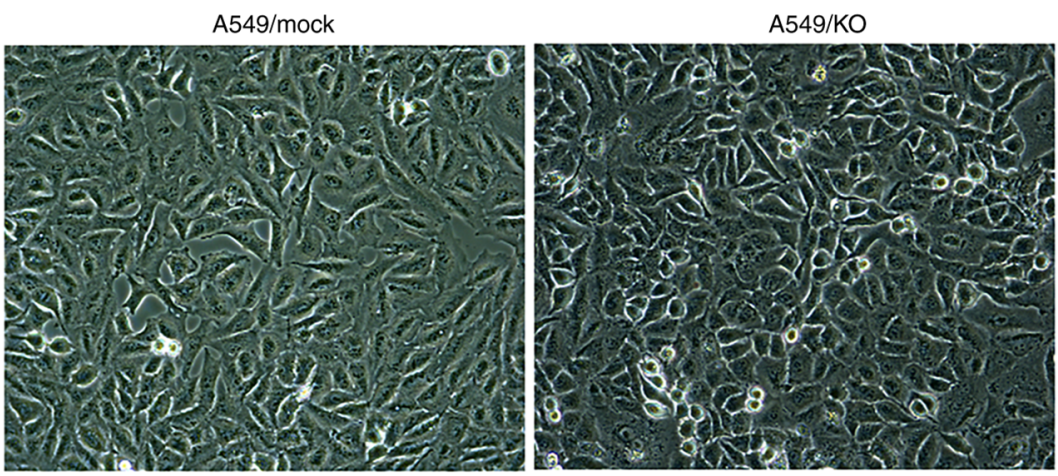

C

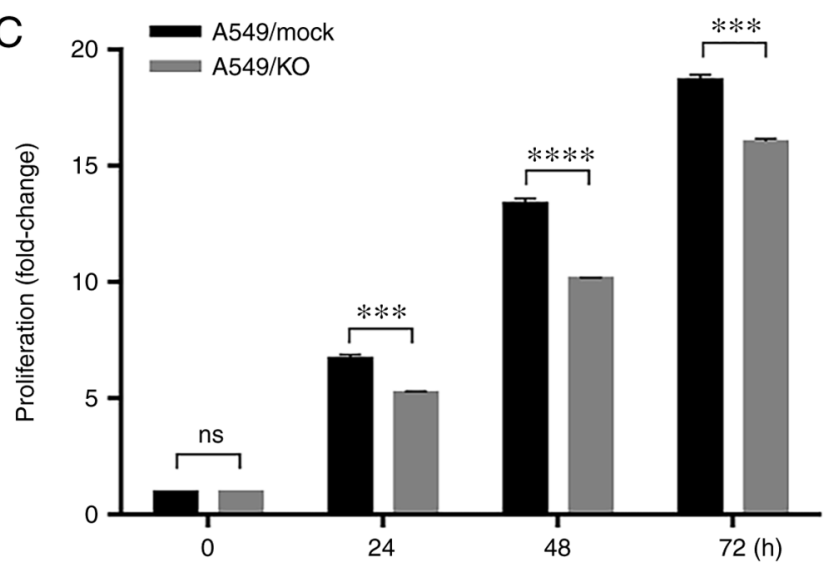

$\mathrm{D}$

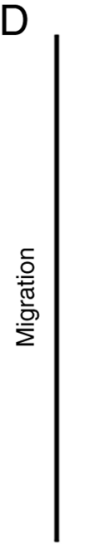

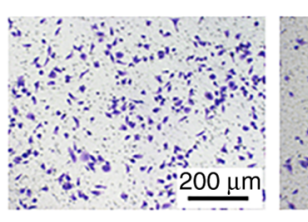
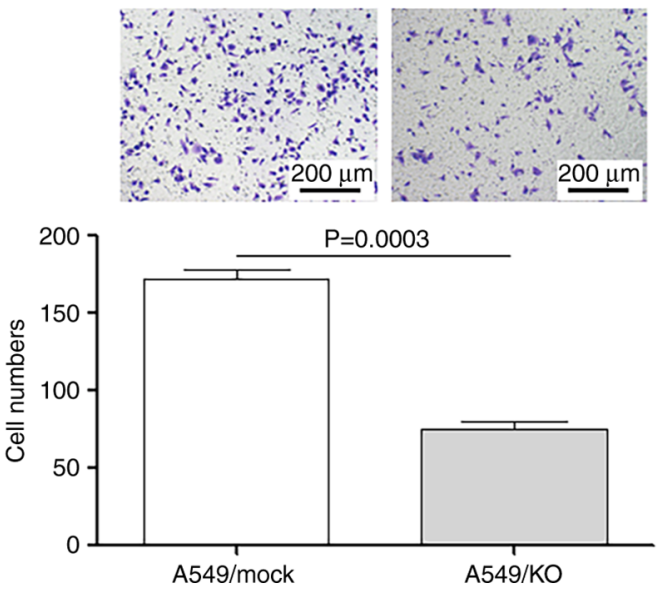

E

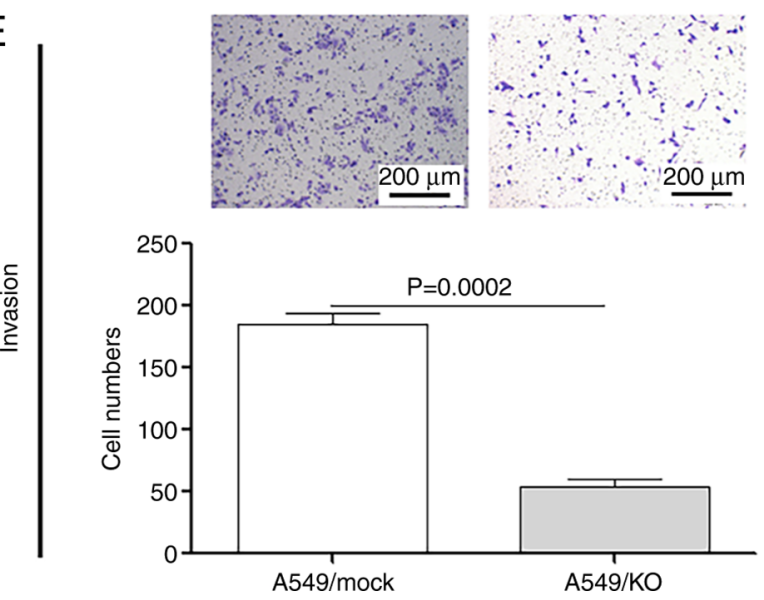

Figure 1. CRISPR/Cas9-mediated $B 7-H 3 \mathrm{KO}$ reduces the proliferation, migration and invasion of A549 cells. (A) Representative western blot (upper) and reverse transcription-quantitative PCR (lower) analysis for B7-H3 expression in B7-H3 KO and mock A549 cells. (B) The microscopic images of B7-H3 mock (left) and $\mathrm{KO}$ (right) A549 cells (magnification, x200). (C) The proliferation of $B 7-H 3 \mathrm{KO}$ and mock A549 cells was determined by WST-8/Cell Counting Kit-8 at $0,24,48$ and $72 \mathrm{~h}$ after cell culturing. Cell proliferation at $0 \mathrm{~h}$ was normalized to $1.0{ }^{* * * *} \mathrm{P}<0.001,{ }^{* * * * *} \mathrm{P}<0.0001$. (D) Migration and (E) invasion of $B 7-H 3 \mathrm{KO}$ and mock A549 cells were assessed by Transwell system as indicated. Data are representative of three independent experiments. ns, no significance; KO, knockout.

compared with those measured upon treatment with DMSO (Fig. 5C-E). Taken together, these results suggested that B7-H3 regulates SIRT1 via the PI3K/AKT pathway in A549 cells.

\section{Discussion}

Data from the SEER Cancer Statistics Review (25) showed that only $\sim 16 \%$ of all NSCLC cases diagnosed between the years 2009 and 2015 were of localized disease, whereas $22 \%$ were diagnosed with regional involvement and 57\% of the cases exhibited dissemination to distant metastasis.
By contrast, the 5-year relative survival rates of localized, regional and distant NSCLC were 57.4, 30.8 and 5.2\%, respectively (25). Therefore, developing an understanding of metastases is critical, since unmanageable migration and invasiveness are responsible for $\sim 90 \%$ of cases of death attributable to NSCLC. In the present study, in vitro evidence has been provided to support that $\mathrm{B} 7-\mathrm{H} 3$ may be closely associated with the aggressive status of NSCLC, since $B 7-H 3$ ablation reduced the numbers of migrating and invading cells by $>50$ and $70 \%$, respectively. This result was consistent with the findings of Yu et al (16), who had previously shown in 

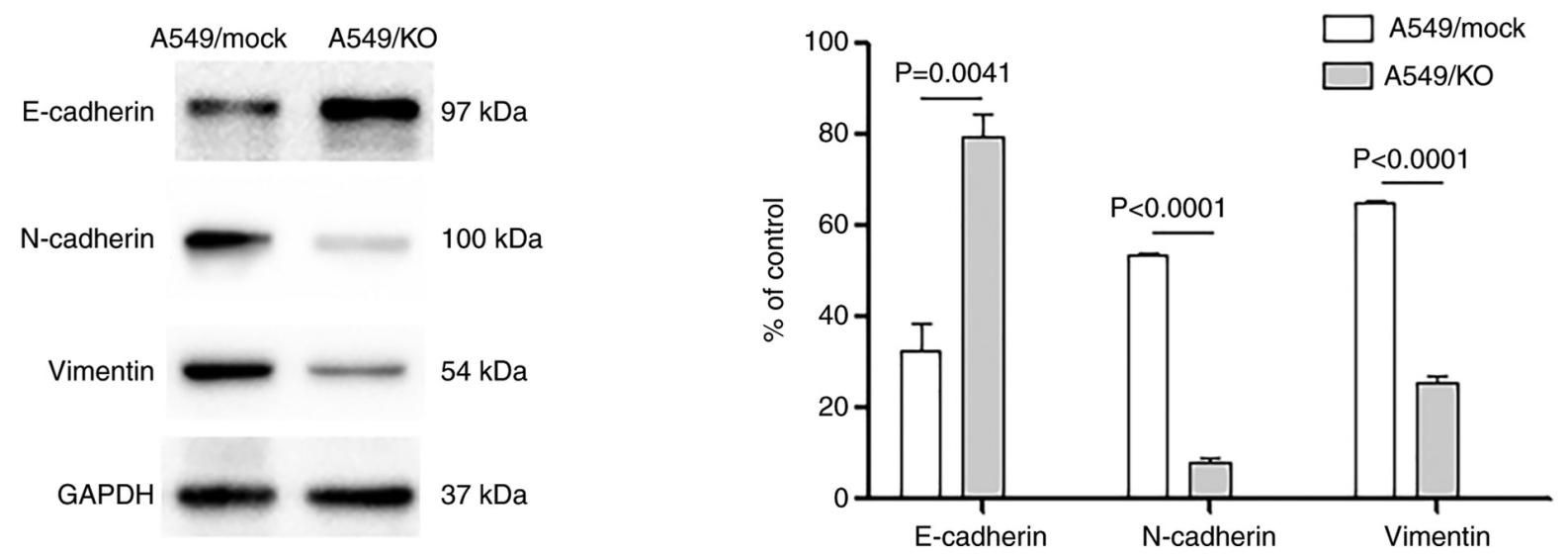

Figure 2. B7- $\mathrm{H} 3 \mathrm{KO}$ regulates epithelial-mesenchymal transition-associated markers of A549 cells. E-cadherin, vimentin and N-cadherin in B7- $H 3$ KO and mock A549 cells were analyzed by western blotting. GAPDH was used as the loading control, data are representative of three independent experiments. KO, knockout.
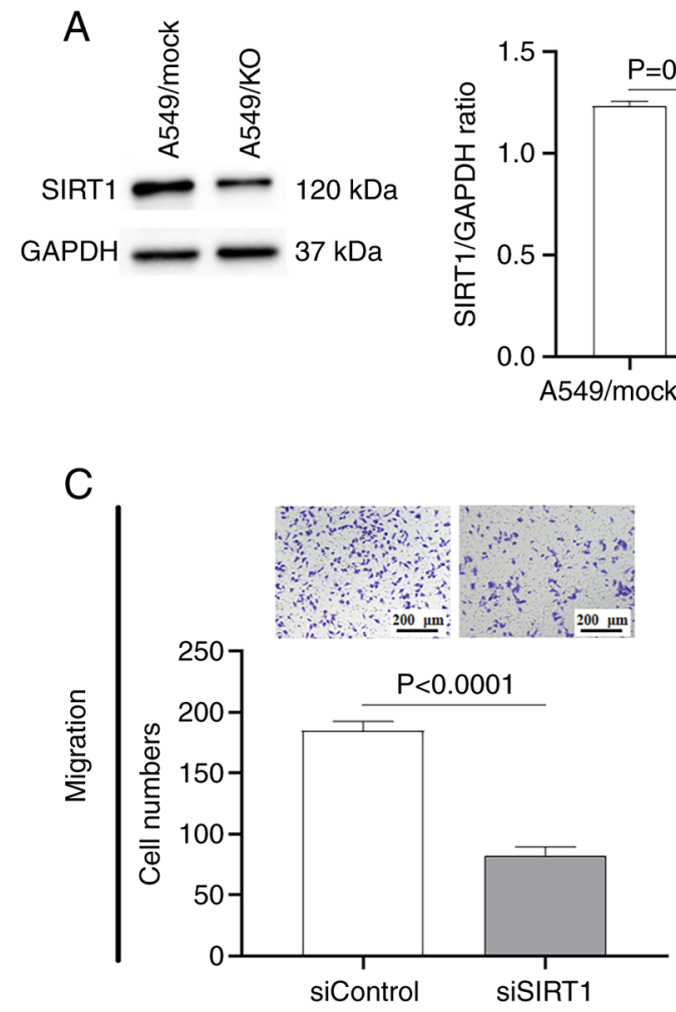
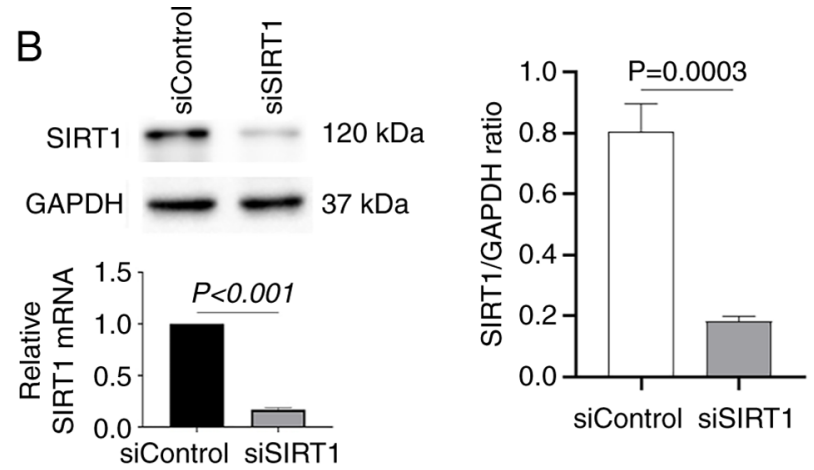

D

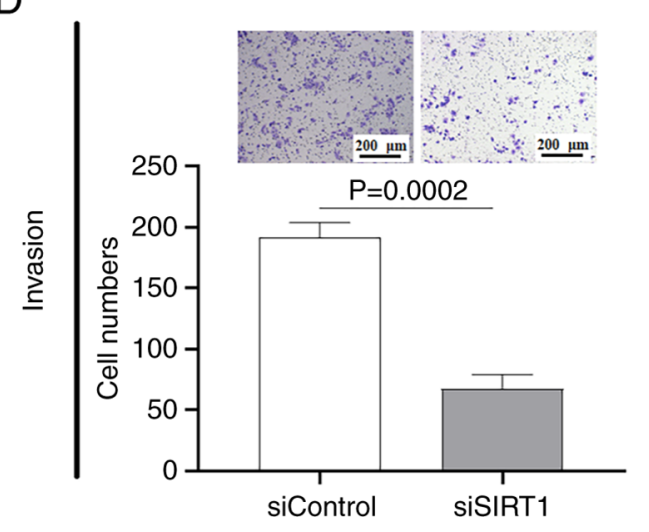

Figure 3. SIRT1 is involved in B7-H3-mediated epithelial-mesenchymal transition activation in A549 cells. (A) Western blot analysis for SIRT1 expression in B7-H3 KO and mock cells. (B) Western blotting (upper left) and the band intensity analysis (right), and reverse transcription-quantitative PCR (lower left) analysis for SIRT1 expression in siSIRT1 and siControl A549 cells as indicated. (C) Migration and (D) invasion of siSIRT1 and siControl A549 cells were assessed by Transwell system. Data are representative of three independent experiments. SIRT1, Sirtuin 1; KO, knockout; si, small interfering RNA.

in vitro experiments the pro-metastasis function of $\mathrm{B} 7-\mathrm{H} 3$ in A549 cells through the siRNA silencing method. The significant effects of B7-H3 on metastases have likewise been demonstrated in cervical cancer (26), bladder cancer (27) and gastric adenocarcinoma cells (28). In vitro B7-H3 knockdown via RNA interference in gastric adenocarcinoma cells led to decreased rates of cell migration and Transwell invasion by $\leq 50 \%$ (28). Collectively, these results highlighted an important role for B7-H3 in promoting cancer cell metastasis, with life-threatening consequences.
The invasiveness and metastatic activity acquired during tumor progression have been shown to be due to EMT. After activation of EMT, tumor cells either lose, or have a reduced expression level of, epithelial-specific E-cadherin, whereas the expression levels of $\mathrm{N}$-cadherin and vimentin are increased as the cells gain mesenchymal activities (6). The results from the present study showed that $B 7-H 3$ deletion led to a substantial suppression of vimentin and $\mathrm{N}$-cadherin expression. By contrast, B7-H3 KO significantly increased the expression of E-cadherin compared with the mock control A549 cells. This result was 

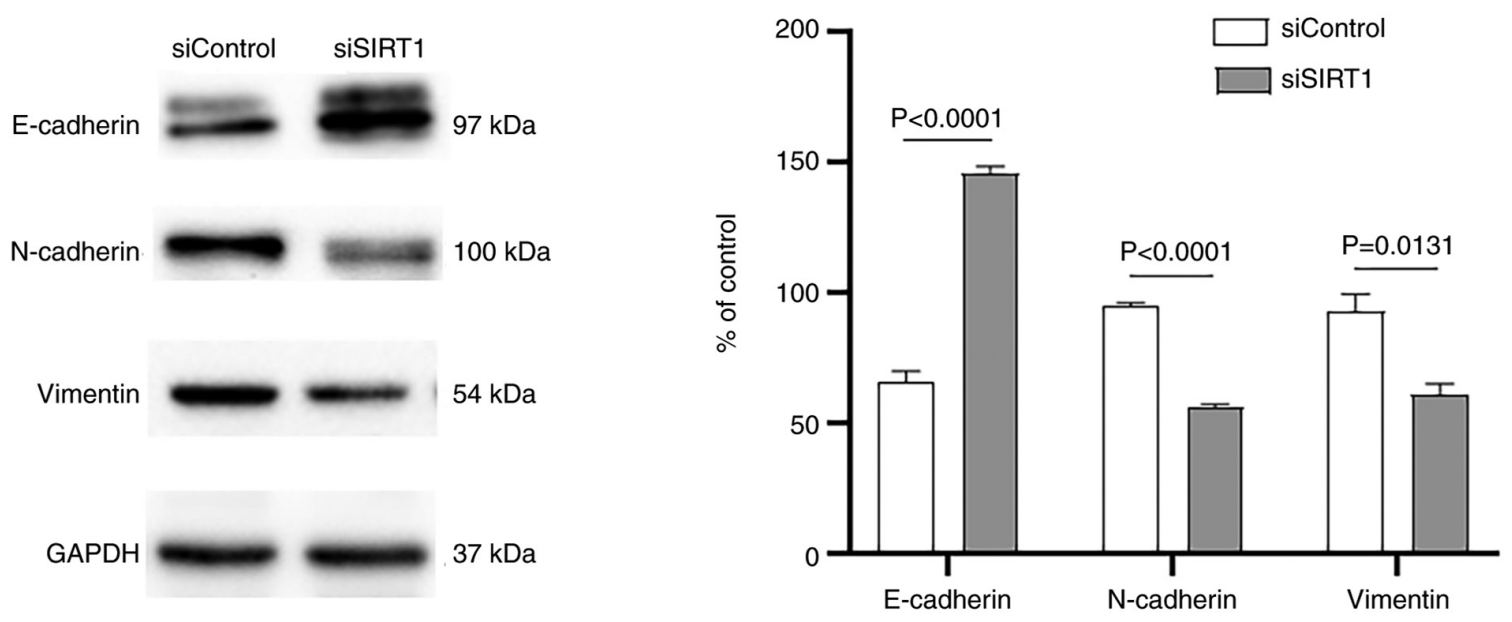

Figure 4. SIRT1 knockdown regulates epithelial-mesenchymal transition-associated markers of A549 cells. E-cadherin, vimentin and N-cadherin expression in siSIRT1 and siControl A549 cells were determined by western blot analysis. GAPDH was used as the loading control, and data are representative of three independent experiments. SIRT1, Sirtuin 1; si, small interfering RNA.
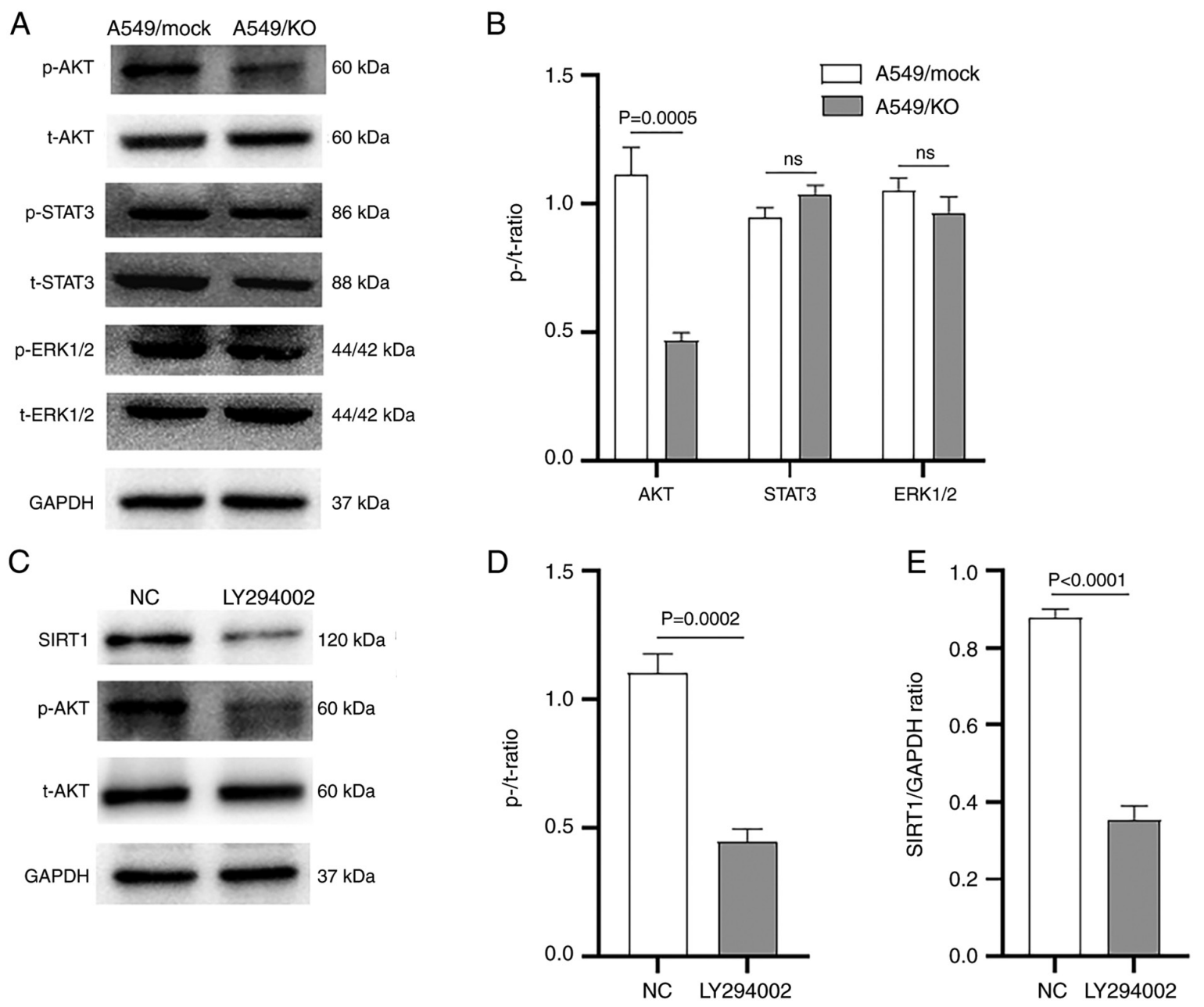

Figure 5. B7-H3 regulates SIRT1 expression via the PI3K/AKT signaling pathway. (A and B) Western blot and band intensity analysis for the t- and p-AKT, STAT3 and ERK1/2 in B7-H3 KO and mock A549 cells. (C-E) Western blot and band intensity analysis for t-AKT, p-AKT and SIRT1 expression in A549 cells treated with LY294002 $(50 \mu \mathrm{M})$. GAPDH was used as the loading control, and relative phosphorylation level (p-/t-) was determined. Data are representative of three independent experiments. SIRT1, Sirtuin 1; t-, total; p-, phosphorylated; KO, knockout; ns, no significance; NC, negative control.

consistent with observations from a previous study (16), further demonstrating that $B 7-H 3$ deletion decreases the EMT process by hindering the loss of adhesive junctions and the gain of mesenchymal activities. The promotion of tumor aggressiveness and invasion resulting from B7-H3 targeting the EMT has also been reported in glioma (18), hepatocellular carcinoma (19), 


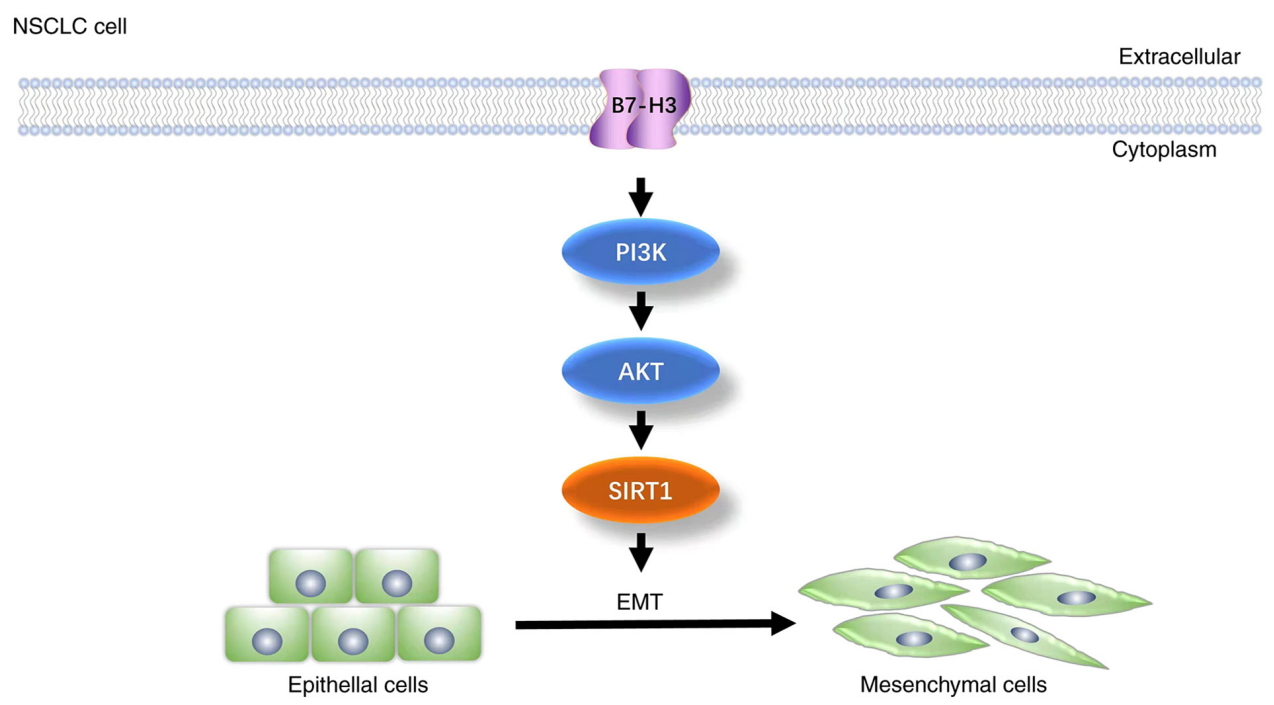

Figure 6. Schematic representation of B7-H3 effects in EMT of lung adenocarcinoma. B7-H3-induced signaling upregulates SIRT1 through the PI3K/AKT pathway, thereby promoting EMT activation of lung adenocarcinoma cells. SIRT1, Sirtuin 1; EMT, epithelial-mesenchymal transition.

cervical cancer (29) and salivary gland adenoid cystic carcinoma (30). As a result of the cells acquiring infiltrating and metastasizing properties, high expression levels of B7-H3 have been shown to be closely associated with poor prognosis of NSCLC (13). Therefore, as with programmed death-ligand 1 (31), B7-H3 is an attractive target for cancer immunotherapy of NSCLC. However, the current strategies available are limited due to the utilization of B7-H3 as an antibody-based tumor antigen (32). Alternative strategies based on non-immunological pro-tumorigenic functions of $\mathrm{B} 7-\mathrm{H} 3$, such as migration and invasion, are in need of further development, since B7-H3-induced signaling in cancer cells remains poorly understood. Thus, the in vivo experimentation to evaluate migration and invasion in animal models, e.g., through magnetic resonance imaging tracking is the focus of future research (33).

In the present study, it was shown that $B 7-H 3$ ablation downregulated SIRT1 expression in A549 cells. Furthermore, SIRT1 silencing produced similar effects to those of $B 7-H 3$ deletion, reducing the numbers of migrating and invasive A549 cells by $>50$ and $60 \%$, respectively. SIRT1 silencing also caused a substantial decrease in the expression levels of vimentin and $\mathrm{N}$-cadherin, whereas E-cadherin was observed to be upregulated in comparison with the scrambled siControl. Therefore, these results suggested that SIRT1 may be involved in the B7-H3-induced EMT process. A recent study has highlighted that SIRT1 is a key regulator of cancer metastasis by promoting EMT, since the dysregulation of SIRT1 substantially altered the in vitro migration ability of cancer cells, and in vivo tumor metastasis (9). In NSCLC, experiments using both SIRT1-targeted miR-138 and its 'sponge', the circular RNA hsa_circ_0006571, mutually confirmed the potential role of SIRT1 in promoting EMT $(34,35)$. However, a study by Li et al (36) demonstrated that SIRT1 could serve a role as a tumor suppressor in NSCLC through attenuating osteopontin-induced NF- $\mathrm{B}$ p 65 acetylation and the EMT. Given that osteopontin is an extracellular matrix protein secreted by both tumor cells and non-tumor stromal cells, it is likely that the observable effects of SIRT1 on EMT are associated with the tumor microenvironment. More recently, in studying colorectal carcinoma, Meng et al (37) presented results that demonstrated alterations in the SIRT1/NF- $\mathrm{B} / \mathrm{B} 7-\mathrm{H} 3 / \mathrm{TNF}-\alpha$ signaling axis, suggesting that the protein level of $\mathrm{B} 7-\mathrm{H} 3$ is downregulated via SIRT1-mediated NF- $\kappa \mathrm{B}$ deactivation. However, whether a negative feedback cycle exists between B7-H3 and SIRT1 requires further investigation. More recently, Yu et al (38) demonstrated that there was a fibroblast growth factor 21-induced SIRT1/PI3K/AKT signaling pathway in A549 cells that could promote cell growth and migration. Thus, positive feedback may also occur to increase the output by mutual promotion between PI3K/AKT and SIRT1.

The results of the present study demonstrated that the PI3K/AKT signaling pathway, but not the JAK2/STAT3 and Raf/MEK/ERK1/2 pathways, was functionally affected by B7-H3 in A549 cells. Furthermore, treatment with the specific PI3K inhibitor LY294002 resulted in significantly decreased SIRT1 and p-AKT expression levels, which suggested that B7-H3 regulates SIRT1 via the PI3K/AKT pathway. The finding that $\mathrm{B} 7-\mathrm{H} 3$ promotes cell migration and invasion (39), drug resistance $(20,40)$ and aerobic glycolysis (21) via the PI3K/AKT pathway has also been reported in ovarian cancer (20), oral squamous carcinoma (21), bladder cancer (39) and colorectal cancer cells (40). On the other hand, B7-H3 has been shown to promote growth and invasion of multiple myeloma (17), glioma (18) and hepatocellular carcinoma (19) by activating the JAK2/STAT3 signaling pathway, and to promote the angiogenesis of colorectal cancer through activating the NF- $\kappa \mathrm{B}$ pathway (41). Therefore, it is likely that B7-H3-induced signaling is transduced through diverse signaling cascades for the purpose of fulfilling several different functions. Tumoral B7-H3 has multiple effects in tumors, by promoting cancer invasion, migration, angiogenesis, drug sensitivity and the Warburg effect. The other possibility is that the signaling cascades downstream of B7-H3 operate differently according to the different cancer types and subtypes. It should be noted that our previous study demonstrated that B7-H3-induced signaling is dissimilar, comparing among lung adenocarcinoma cell lines with divergent epidermal growth factor receptor mutation patterns (23). 
Taken together, the present results have confirmed that B7-H3 contributes to the migration and invasion of NSCLC cells by promoting the EMT process. The present study has provided evidence that the class III histone deacetylase SIRT1 may be involved in B7-H3-induced EMT, and also that B7-H3 regulates SIRT1 via the PI3K/AKT signaling pathway in NSCLC A549 cells (Fig. 6). These findings may prove to be useful in terms of identifying novel strategies for therapeutic intervention in NSCLC metastasis. Further studies are required, however, to provide in vivo evidence of the effectiveness and therapeutic feasibility of targeting B7-H3-mediated EMT in NSCLC.

\section{Acknowledgements}

Not applicable.

\section{Funding}

This work was funded by the Natural Science Foundation of Anhui Province (grant no. 1808085MH229) and Key Research and Development Program of Anhui Province (grant no. 202004j07020027).

\section{Availability of data and materials}

The datasets used and/or analyzed during the current study are available from the corresponding author on reasonable request.

\section{Authors' contributions}

LC and HL designed the present study and analyzed the data. HL and MD performed the experiments and drafted the article. NZ and YY contributed to the analysis and interpretation of all data. LC revised the manuscript and performed the manuscript submission. HL and MD confirm the authenticity of all the raw data. All authors have read and approved the final manuscript.

\section{Ethics approval and consent to participate}

Not applicable.

\section{Patient consent for publication}

Not applicable.

\section{Competing interests}

The authors declare that they have no competing interests.

\section{References}

1. Bray F, Ferlay J, Soerjomataram I, Siegel RL, Torre LA and Jemal A: Global cancer statistics 2018: GLOBOCAN estimates of incidence and mortality worldwide for 36 cancers in 185 countries. CA Cancer J Clin 68: 394-424, 2018.

2. Travis WD, Brambilla E, Burke AP, Marx A and Nicholson AG: Introduction to the 2015 World Health Organization Classification of tumors of the lung, pleura, thymus, and heart. J Thorac Oncol 10: 1240-1242, 2015.
3. Huang Z, Su W, Lu T, Wang Y, Dong Y, Qin Y, Liu D, Sun L and Jiao W: First-line immune-checkpoint inhibitors in non-small cell lung cancer: Current landscape and future progress. Front Pharmacol 11: 578091, 2020.

4. Mittal V: Epithelial mesenchymal transition in tumor metastasis. Annu Rev Pathol 13: 395-412, 2018.

5. Huang W, Yan Y, Liu Y, Lin M, Ma J, Zhang W, Dai J, Li J, Guo Q, Chen $\mathrm{H}$, et al: Exosomes with low miR-34c-3p expression promote invasion and migration of non-small cell lung cancer by upregulating integrin $\alpha 2 \beta 1$. Signal Transduct Target Ther 5: 39, 2020.

6. Jørgensen CLT, Forsare C, Bendahl PO, Falck AK, Fernö M, Lövgren K, Aaltonen K and Rydén L: Expression of epithelial-mesenchymal transition-related markers and phenotypes during breast cancer progression. Breast Cancer Res Treat 181: 369-381, 2020

7. Sauve AA, Wolberger C, Schramm VL and Boeke JD: The biochemistry of sirtuins. Annu Rev Biochem 75: 435-465, 2006.

8. Zhao B, Li X, Zhou L, Wang Y and Shang P: SIRT1: A potential tumour biomarker and therapeutic target. J Drug Target 27: 1046-1052, 2019.

9. Choupani J, Mansoori DS, Bayat S, Alivand MR and Shekari KM: Narrower insight to SIRT1 role in cancer: A potential therapeutic target to control epithelial-mesenchymal transition in cancer cells. J Cell Physol 233: 4443-457, 2018

10. Nehama D, Di Ianni N, Musio S, Du H, Patane M, Pollo B, Finocchiaro G, Park JJ, Dunn DE, Edwards DS, et al: B7-H3-redirected chimeric antigen receptor T cells target glioblastoma and neurospheres. EBioMedicine 47: 33-43, 2019.

11. Zhang Z, Jiang C, Liu Z, Yang M, Tang X, Wang Y, Zheng M, Huang J, Zhong K, Zhao S, et al: B7-H3-targeted CAR-T cells exhibit potent antitumor effects on hematologic and solid tumors. Mol Ther Oncolytics 17: 180-189, 2020.

12. Seaman S, Zhu Z, Saha S, Zhang XM, Yang MY, Hilton MB, Morris K, Szot C, Morris H, Swing DA, et al: Eradication of tumors through simultaneous ablation of CD276/B7-H3-positive tumor cells and tumor vasculature. Cancer Cell 31: 501-515, 2017.

13. Altan M, Pelekanou V, Schalper KA, Toki M, Gaule P, Syrigos K, Herbst RS and Rimm DL: B7-H3 expression in NSCLC and its association with B7-H4, PD-L1 and tumor-infiltrating lymphocytes. Clin Cancer Res 23: 5202-5209, 2017.

14. Yonesaka K, Haratani K, Takamura S, Sakai H, Kato R, Takegawa N, Takahama T, Tanaka K, Hayashi H, Takeda M, et al: B7-H3 negatively modulates CTL-mediated cancer immunity. Clin Cancer Res 24: 2653-2664, 2018.

15. Cai D, Li J, Liu D, Hong S, Qiao Q, Sun Q, Li P, Lyu N, Sun T, Xie S, et al: Tumor-expressed B7-H3 mediates the inhibition of antitumor T-cell functions in ovarian cancer insensitive to PD-1 blockade therapy. Cell Mol Immunol 17: 227-236, 2020.

16. Yu TT, Zhang T, Lu X and Wang RZ: B7-H3 promotes metastasis, proliferation, and epithelial-mesenchymal transition in lung adenocarcinoma. Onco Targets Ther 11: 4693-4700, 2018.

17. Lin L, Cao L, Liu Y, Wang K, Zhang X, Qin X, Zhao D, Hao J, Chang Y, Huang X, et al: B7-H3 promotes multiple myeloma cell survival and proliferation by ROS-dependent activation of Src/STAT3 and c-Cbl-mediated degradation of SOCS3. Leukemia 33: 1475-1486, 2019.

18. Zhong C, Tao B, Chen Y, Guo Z, Yang X, Peng L, Xia X and Chen L: B7-H3 Regulates Glioma growth and cell invasion through a JAK2/STAT3/Slug-dependent signaling pathway. Onco Targets Ther 13: 2215-2224, 2020.

19. Kang FB, Wang L, Jia HC, Li D, Li HJ, Zhang YG and Sun DX: B7-H3 promotes aggression and invasion of hepatocellular carcinoma by targeting epithelial-to-mesenchymal transition via JAK2/STAT3/Slug signaling pathway. Cancer Cell Int 15: 45, 2015.

20. Zhou L and Zhao Y: B7-H3 induces ovarian cancer drugs resistance through an PI3K/AKT/BCL-2 signaling pathway. Cancer Manag Res 11: 10205-10214, 2019

21. Li Z, Liu J, Que L and Tang X: The immunoregulatory protein B7-H3 promotes aerobic glycolysis in oral squamous carcinoma via PI3K/Akt/mTOR pathway. J Cancer 10: 5770-5784, 2019.

22. Flem-Karlsen K, Fodstad $\varnothing$ and Nunes-Xavier CE: B7-H3 immune checkpoint protein in human cancer. Curr Med Chem 27: 4062-4086, 2020.

23. Ding M, Liao H, Zhou N, Yang Y, Guan S and Chen L: B7-H3-induced signaling in lung adenocarcinoma cell lines with divergent epidermal growth factor receptor mutation patterns. Biomed Res Int 2020: 8824805, 2020.

24. Livak KJ and Schmittgen TD: Analysis of relative gene expression data using real-time quantitative PCR and the 2(-Delta Delta C(T)) method. Methods 25: 402-408, 2001. 
25. Howlader N, Noone AM, Krapcho M, Miller D, Brest A, Yu M Ruhl J, Tatalovich Z, Mariotto A, Lewis DR, et al (eds): SEER Cancer Statistics Review, 1975-2016, National Cancer Institute. https://seer.cancer.gov/csr/1975_2016/. Bethesda, MD, 2019.

26. Li Y, Zhang J, Han S, Qian Q, Chen Q, Liu L and Zhang Y: B7-H3 promotes the proliferation, migration and invasiveness of cervical cancer cells and is an indicator of poor prognosis. Oncol Rep 38: 1043-1050, 2017.

27. Xu ZL, Zhang Y, Wang L, Li F, Man HW, Li PF and Shan BE: B 7-H3 promotes malignant progression of muscle-invasive bladder cancer. Oncol Rep 40: 2722-2733, 2018.

28. Dai W, Shen G, Qiu J, Zhao X and Gao Q: Aberrant expression of B7-H3 in gastric adenocarcinoma promotes cancer cell metastasis. Oncol Rep 32: 2086-2092, 2014.

29. Yang X, Feng KX, Li H, Wang L and Xia H: MicroRNA-199a inhibits cell proliferation, migration, and invasion and activates AKT/mTOR signaling pathway by targeting B7-H3 in cervical cancer. Technol Cancer Res Treat: Aug 28, 2020 (Epub ahead of print). doi: $10.1177 / 1533033820942245$.

30. Fan TF, Deng WW, Bu LL, Wu TF, Zhang WF and Sun ZJ: B7-H3 regulates migration and invasion in salivary gland adenoid cystic carcinoma via the JAK2/STAT3 signaling pathway. Am J Transl Res 9: 1369-1380, 2017

31. Luo M, Wang F, Zhang H, To KKW, Wu S, Chen Z, Liang S and Fu L: Mitomycin C enhanced the efficacy of PD-L1 blockade in non-small cell lung cancer. Signal Transduct Target Ther 5: 141, 2020 .

32. Kontos F, Michelakos T, Kurokawa T, Sadagopan A, Schwab JH, Ferrone CR and Ferrone S: B7-H3: An attractive target for antibody-based immunotherapy. Clin Cancer Res 27: 1227-1235, 2021.

33. Bretschi M, Merz M, Komljenovic D, Berger MR, Semmler W and Bäuerle T: Cilengitide inhibits metastatic bone colonization in a nude rat model. Oncol Rep 26: 843-851, 2011.
34. Ye Z, Fang B, Pan J, Zhang N, Huang J, Xie C, Lou T and Cao Z: miR-138 suppresses the proliferation, metastasis and autophagy of non-small cell lung cancer by targeting Sirt1. Oncol Rep 37: 3244-3252, 2017

35. Wang HL, Wang HR, Liang Y, Hu AN, Enguita FJ, Zhou XG and Dong J: Hsa_circ_0006571 promotes spinal metastasis through sponging microRNA-138 to regulate sirtuin 1 expression in lung adenocarcinoma. Transl Lung Cancer Res 9: 2411-2427, 2020.

36. Li X, Jiang Z, Li X and Zhang X: SIRT1 overexpression protects non-small cell lung cancer cells against osteopontin-induced epithelial-mesenchymal transition by suppressing $\mathrm{NF}-\kappa \mathrm{B}$ signaling. Onco Targets Ther 11: 1157-1171, 2018.

37. Meng F, Yang M, Chen Y, Chen W and Wang W: miR-34a induces immunosuppression in colorectal carcinoma through modulating a SIRT1/NF- $\kappa$ B/B7-H3/TNF- $\alpha$ axis. Cancer Immunol Immunother 70: 2247-2259, 2021.

38. Yu X, Li Y, Jiang G, Fang J, You Z, Shao G, Zhang Z, Jiao A and Peng X: FGF21 promotes non-small cell lung cancer progression by SIRT1/PI3K/AKT signaling. Life Sci 269: 118875, 2021.

39. Li Y, Guo G, Song J, Cai Z, Yang J, Chen Z, Wang Y, Huang Y and Gao Q: B7-H3 promotes the migration and invasion of human bladder cancer cells via the PI3K/Akt/STAT3 signaling pathway. J Cancer 8: 816-824, 2017.

40. Zhang P, Chen Z, Ning K, Jin J and Han X: Inhibition of B7-H3 reverses oxaliplatin resistance in human colorectal cancer cells. Biochem Biophys Res Commun 490: 1132-1138, 2017.

41. Wang R, Ma Y, Zhan S, Zhang G, Cao L, Zhang X, Shi T and Chen W: B7-H3 promotes colorectal cancer angiogenesis through activating the NF- $\kappa \mathrm{B}$ pathway to induce VEGFA expression. Cell Death Dis 11: 55, 2020.

This work is licensed under a Creative Commons Attribution-NonCommercial-NoDerivatives 4.0 International (CC BY-NC-ND 4.0) License. 